\title{
Optical Counterpart of the X-ray Transient RX J0117·6-7330: Spectroscopy and Photometry
}

\author{
Roberto Soria \\ Mount Stromlo Observatory, Private Bag, Weston Creek PO, \\ Weston Creek, ACT 2611, Australia \\ roberto@mso.anu.edu.au \\ Received 1998 October 12, accepted 1999 February 20
}

\begin{abstract}
We conducted spectroscopic and photometric observations of the optical companion of the X-ray transient RX J0117·6-7330 in the Small Magellanic Cloud, during a quiescent state. The primary star is identified as a B0.5 IIIe with a mass $M_{*}=(18 \pm 2) M_{\odot}$ and bolometric magnitude $M_{\mathrm{bol}}=-7 \cdot 4 \pm 0 \cdot 2$. The main spectral features are strong $\mathrm{H} \alpha$ emission, $\mathrm{H} \beta$ and $\mathrm{H} \gamma$ emission cores with absorption wings, and narrow $\mathrm{He}_{\mathrm{I}}$ and $\mathrm{O}_{\text {II }}$ absorption lines. Equivalent widths and full widths at half maximum of the main lines are listed. The average systemic velocity over our observing run is $v_{r}=(184 \pm 4) \mathrm{km} \mathrm{s}^{-1}$; measurements over a longer period of time are needed to determine the binary period and the $K$ velocity of the primary. We determine a projected rotational velocity $v \sin i=(145 \pm 10) \mathrm{km} \mathrm{s}^{-1}$ for the Be star; if we assume a true rotational velocity at the equator $v=(400 \pm 50) \mathrm{km} \mathrm{s}^{-1}$, we deduce that the inclination angle of the system is $i=(21 \pm 3) \mathrm{deg}$.
\end{abstract}

Keywords: galaxies: Magellanic Clouds — stars: individual (RX J0117·6-7330) — X-ray: binaries

\section{Introduction}

The X-ray transient RX J0117·6-7330 was discovered by the Position Sensitive Proportional Counter on board ROSAT on 1-2, October 1992 (Clark, Remillard \& Woo 1996, 1997); it is located approximately $5^{\prime}$ southeast of the X-ray binary SMC X-1. Its optical counterpart was observed by Charles, Southwell \& O'Donoghue (1996), and by Clark, Remillard \& Woo (1997), and has the characteristics of a Be star; the system is therefore likely to be a high-mass X-ray binary (HMXB).

Systems with a Be-type primary are the largest group among the HMXBs, both in the Galaxy and in the Magellanic Clouds (van Paradijs \& McClintock 1995; Kahabka \& Pietsch 1996). In these systems, X-ray outbursts lasting from weeks to months are caused by sudden enhancements of the equatorial mass loss of the Be star; during an active state, a modulation corresponding to the orbital period is often observed, with increased X-ray emission when the compact object transits near periastron in an eccentric orbit (van den Heuvel \& Rappaport 1987). Active states are separated by longer inactive periods, often lasting several years.

The nature of the compact object in RX J0117.6 - 7330 is not yet known; its soft X-ray spectrum, its long decay time after the 1992 outburst and the absence of pulsations make this system a possible black-hole candidate (Clark, Remillard \& Woo 1997). A common way to determine whether the compact object in an
X-ray binary can be a neutron star is to deduce its mass function from the radial velocity amplitude of the primary; such measurements are more difficult in HMXBs owing to the low velocity expected for the primary and to the long binary period (tens to hundreds of days).

\section{Observations and Data Analysis}

We observed the optical counterpart of RX J0117 · 6 7330 from 1988 August 20 to August 23, simultaneously with the 40 in telescope (photometry) and the ANU $2 \cdot 3 \mathrm{~m}$ telescope (spectroscopy) at Siding Spring Observatory. Conditions were photometric during the first half of the first night and on the last night.

\subsection{High-resolution Optical Spectroscopy}

Optical spectra of the primary were obtained with the Double Beam Spectrograph on the $2 \cdot 3 \mathrm{~m} \mathrm{ANU}$ telescope at Siding Spring Observatory, with 1200 groove/mm gratings for both the blue (4150-5115 $\AA$ ) and the red (6200-7140 ^) spectral regions (resolution $1 \cdot 2 \AA \mathrm{FWHM}$ ); the detectors used were SiTe $1752 \times$ 532 CCDs in both arms of the spectrograph.

Figures 1 and 2 show the average of seven $600 \mathrm{~s}$ spectra taken on August 20 in photometric conditions, for the blue and the red part of the spectrum respectively. Atmospheric absorption bands at $\lambda>6860 \AA$ have been removed from the red spectrum using the spectra of the calibration star LTT7379. Wavelengths are heliocentric. 


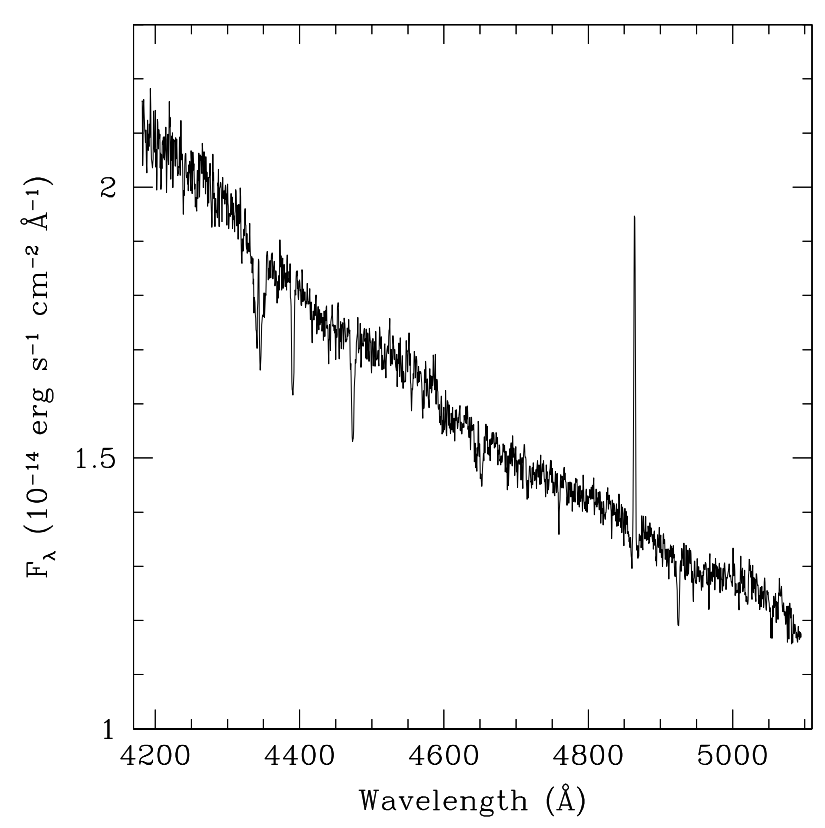

Figure 1-Flux-calibrated blue spectrum of the optical counterpart of RX J0117·6-7330, taken on 1998 August 20, from the ANU $2 \cdot 3 \mathrm{~m}$ telescope at Siding Spring Observatory.

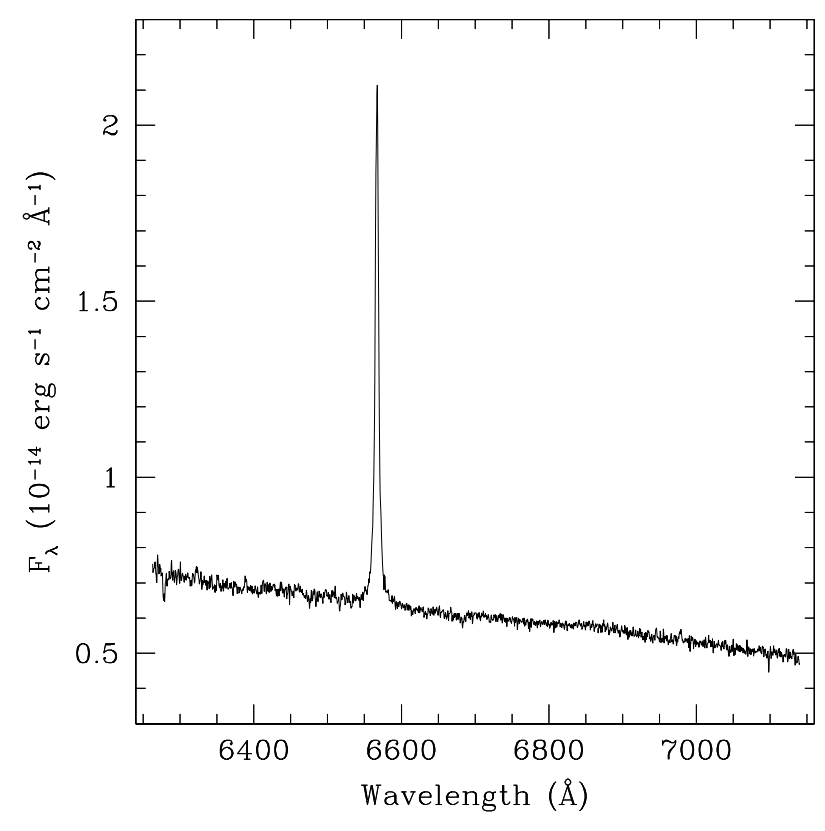

Figure 2-Flux-calibrated red spectrum of the optical counterpart of RX J0117.6-7330, taken on 1998 August 20, from the ANU $2 \cdot 3 \mathrm{~m}$ telescope at Siding Spring Observatory.

The most prominent feature in the blue spectral region is a strong $\mathrm{H} \beta$ emission line: the equivalent width of the emission core, defined as in Dachs et al. $(1981)^{*}$, is $\mathrm{EW}=(-1 \cdot 35 \pm 0 \cdot 05) \AA$; broader photospheric absorption wings are also present. $\mathrm{H} \gamma$ is seen in absorption with a narrower and weaker emission core $[\mathrm{EW}=(-0 \cdot 27 \pm 0 \cdot 02) \AA]$. Narrow absorption is observed from He $\mathrm{I} \lambda 4388$ ( $\mathrm{EW}=0 \cdot 4$ $\AA), \mathrm{He}_{\mathrm{I}} \lambda \lambda 4471,4472(\mathrm{EW}=0 \cdot 6 \AA)$ and He $\mathrm{I} \lambda 4922$ $(\mathrm{EW}=0 \cdot 3 \AA)$. Figures 3,4 and 5 show the region of the blue spectrum (average of all four nights, normalised to the continuum) around $\mathrm{H} \gamma$ and $\mathrm{H} \beta$. Other weaker lines identified in the blue spectrum are listed in Table 1.

In the red spectral region (Figure 6 ) the strongest emission line is $\mathrm{H} \alpha(\mathrm{EW}=-16 \cdot 0 \pm 1 \cdot 0 \AA)$; weaker

Table 1. Most prominent lines found in the optical spectrum of RX J0117·6-7330 (Balmer lines excluded)

\begin{tabular}{lr}
\hline \multicolumn{1}{c}{ Line } & \multicolumn{1}{c}{ EW $(\AA)$} \\
\hline He II $\lambda 4200$ & $0 \cdot 05 \pm 0 \cdot 02$ \\
O II $\lambda 4254$ & $0 \cdot 10 \pm 0 \cdot 02$ \\
O II $\lambda 4317$ & $0 \cdot 10 \pm 0 \cdot 02$ \\
O II $\lambda 4349$ & $0 \cdot 15 \pm 0 \cdot 05$ \\
O II $\lambda 4367$ & $0 \cdot 03 \pm 0 \cdot 01$ \\
He I $\lambda 4388$ & $0 \cdot 40 \pm 0 \cdot 10$ \\
O II $\lambda \lambda 4415,4417$ & $0 \cdot 07 \pm 0 \cdot 02$ \\
He I $\lambda 4438$ & $0 \cdot 08 \pm 0 \cdot 02$ \\
He I $\lambda \lambda 4471,4472$ & $0 \cdot 60 \pm 0 \cdot 10$ \\
S I $\lambda 4550$ & $-0 \cdot 07 \pm 0 \cdot 02$ \\
N II $/$ Si III $\lambda 4553$ & $0 \cdot 10 \pm 0 \cdot 02$ \\
Si II $\lambda 4568$ & $0 \cdot 09 \pm 0 \cdot 02$ \\
O II $\lambda 4642$ & $0 \cdot 15 \pm 0 \cdot 02$ \\
O II $\lambda \lambda 4649,4651$ & $0 \cdot 25 \pm 0 \cdot 05$ \\
O II $\lambda 4662$ & $0 \cdot 08 \pm 0 \cdot 02$ \\
O II $\lambda 4676$ & $0 \cdot 09 \pm 0 \cdot 02$ \\
He II $\lambda 4686$ & $0 \cdot 10 \pm 0 \cdot 02$ \\
He I $\lambda 4713$ & $0 \cdot 12 \pm 0 \cdot 02$ \\
He I $\lambda 4922$ & $0 \cdot 30 \pm 0 \cdot 05$ \\
S II $\lambda 6386$ & $-0 \cdot 20 \pm 0 \cdot 02$ \\
He I $\lambda 6678$ & $0 \cdot 20 \pm 0 \cdot 02$ \\
\hline
\end{tabular}

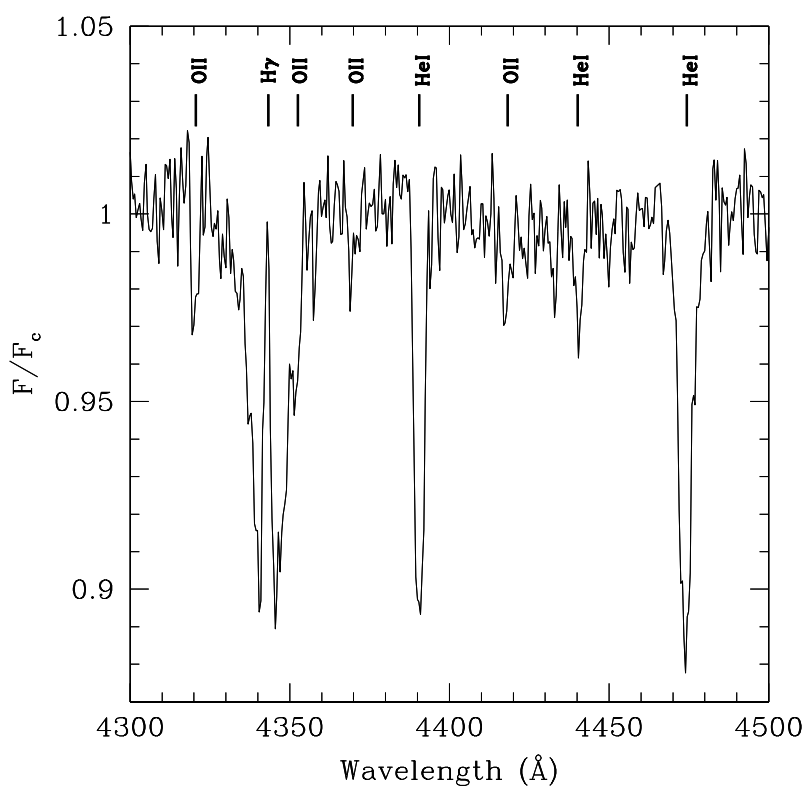

Figure 3-Portion of the co-added blue spectrum normalised to the continuum; some of the absorption lines listed in the text have been identified here.

\footnotetext{
${ }^{*}$ For emission cores in the centre of a surrounding photospheric absorption line, the equivalent width is derived by measuring the area between the normalised emission line profile and a straight line connecting the intensity minima in the absorption wings; see Figure 1 in Dachs et al. (1981).
} 
emission is seen from S II $\lambda 6386(\mathrm{EW}=-0 \cdot 20 \pm 0 \cdot 02$ $\AA)$; He I $\lambda 6678$ is seen in absorption $(\mathrm{EW}=0 \cdot 20 \pm 0 \cdot 02$ A).

Based on these features, the primary star can be identified as a B0.5IIIe, consistently with the results of Charles et al. (1996) and of Clark et al. (1997).

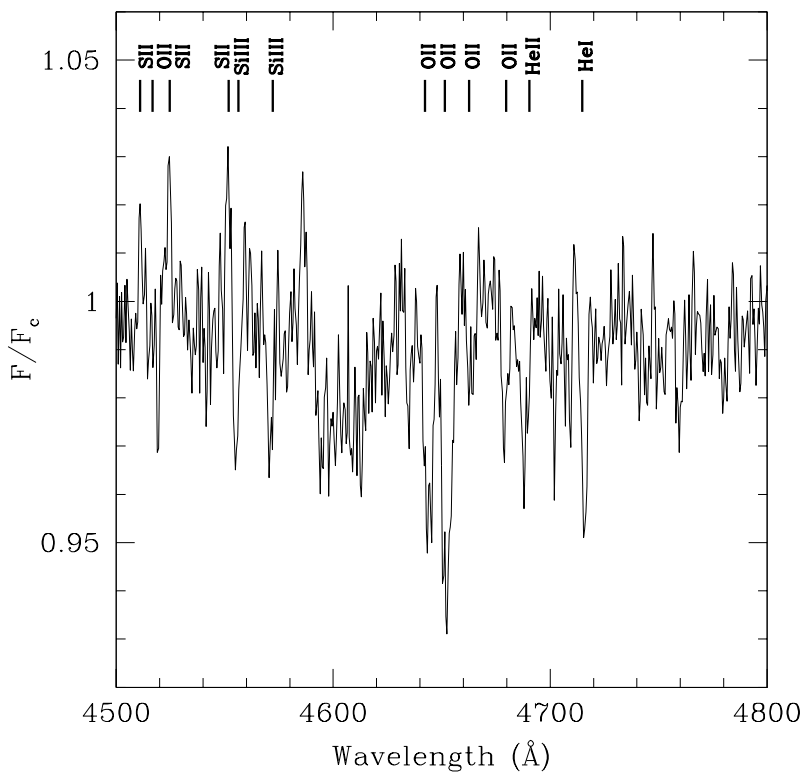

Figure 4-Portion of the co-added blue spectrum normalised to the continuum, with some of the major lines identified. A blend of $\mathrm{O}$ II, $\mathrm{N}$ II and $\mathrm{C}$ II absorption lines is responsible for the broad absorption feature at $\lambda \sim 4600 \AA$.

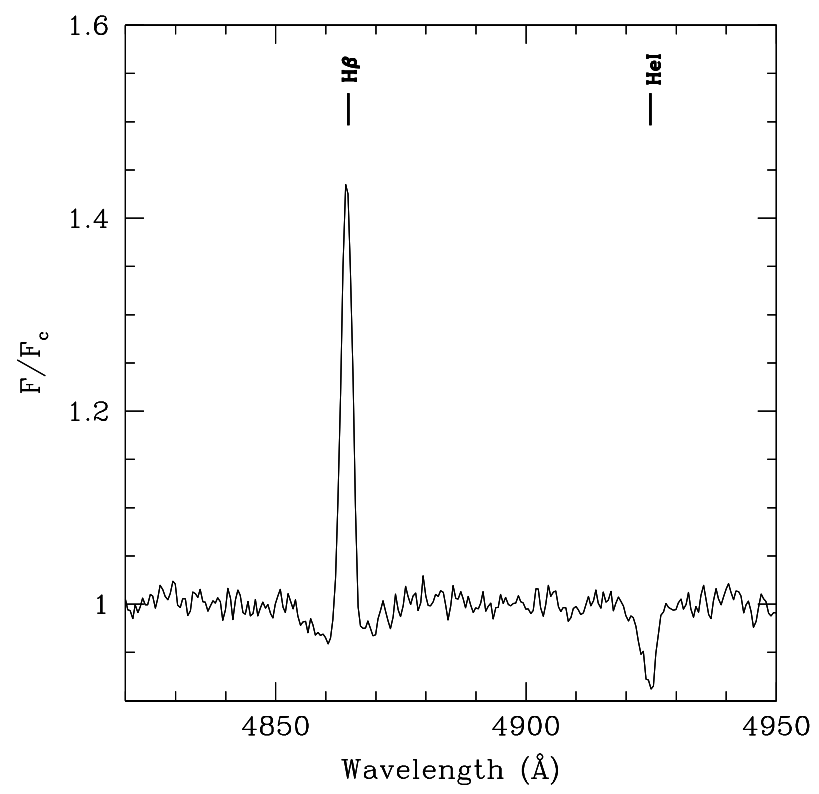

Figure 5-Portion of the co-added blue spectrum, normalised to the continuum. $\mathrm{H} \beta$ and $\mathrm{He}$ I $\lambda 4922$ are the main features in this spectral region.

\subsection{Photometry}

Photometric observations of the system were conducted from the SSO 40 in telescope; the detector used was a SiTe $2048 \times 2048$ CCD. We obtained apparent magnitudes $B=14 \cdot 12 \pm 0 \cdot 01, V=14 \cdot 19 \pm$ $0 \cdot 01, R=14 \cdot 13 \pm 0 \cdot 01$ and $I=14 \cdot 10 \pm 0 \cdot 01$; no significant variations in the brightness of the star were observed during the run. Following Clark et al. (1997) [see also van der Klis et al. (1992)], we have adopted a reddening of $E(B-V)=0 \cdot 08 \pm 0 \cdot 01$; adopting also a distance modulus for the SMC of $d=18 \cdot 9$ (Feast 1991) we get absolute magnitudes $M_{B}=-5 \cdot 11 \pm 0 \cdot 04, M_{V}=-4 \cdot 96 \pm 0 \cdot 03, M_{R}=$ $-4 \cdot 96 \pm 0 \cdot 03$ and $M_{I}=-4 \cdot 92 \pm 0 \cdot 02$.

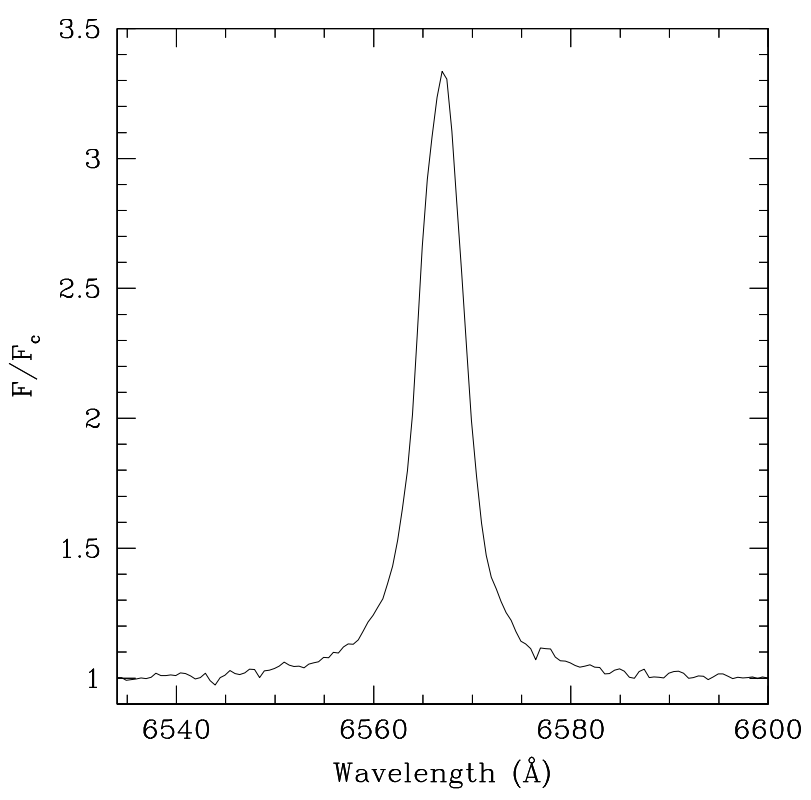

Figure 6- $\mathrm{H} \alpha$ line profile, normalised to the continuum, in the co-added red spectrum. Balmer lines always appear single-peaked, and their profile is quite different from that expected for emission from a thin disk.

We expect the Be star to appear redder than a nonBe star of similar temperature (Bessell 1993) because of the radiation from the circumstellar disk (colder than the star); Paschen continuum emission usually gives a particularly significant contribution in the near IR. Assuming that the $B$ magnitude is the least affected by this additional contribution, and using the theoretical isochrones of Bertelli et al. (1994) in the range of metallicities $Z=0 \cdot 002-0 \cdot 004$ (Bessell 1993), we can estimate a bolometric magnitude $M_{\mathrm{bol}}=-7 \cdot 4 \pm 0 \cdot 2$ and an effective temperature $\log T_{\text {eff }}=4 \cdot 44 \pm 0 \cdot 03$. These values correspond to the giant phase of evolution for stars of mass $M_{*}=(18 \pm 2) M_{\odot}$, and are therefore consistent with the spectral identification of the optical counterpart of RX J0117·6-7330 as a B0·5 IIIe star.

Using the results of Underhill et al. (1979), we can also infer a radius $R_{*} \simeq 10 R_{\odot}$, although values for the same spectral types determined by Popper (1980) are lower by $\sim 30 \%$. 


\subsection{Projected Rotational Velocity and Radial Velocity}

An interesting feature of our spectra is the small full width at half maximum (FWHM) of all the lines; the narrowest absorption lines are He I $\lambda 4388$ and HeI $\lambda 4922$, for both of which we calculate an average $\mathrm{FWHM}=(210 \pm 20) \mathrm{km} \mathrm{s}^{-1}$. The Doppler broadening of spectral lines is a function of the projected rotational velocity $v \sin i$. The FWHM of the He I $\lambda 4471$ absorption line was used by Slettebak et al. (1975) as a parameter for a system of standard rotational velocity stars. We determine an average $\mathrm{FWHM}=(4 \cdot 3 \pm 0 \cdot 3) \AA$ for HeI $\lambda 4471$ in our spectra; correcting for the instrumental broadening (resolution $=1 \cdot 2 \AA$ ), we estimate a $\mathrm{FWHM}=(4 \cdot 1 \pm 0 \cdot 3) \AA=(275 \pm 20)$ $\mathrm{kms}^{-1}$. Comparing this value with those listed in Slettebak et al. (1975) for the same spectral type, we estimate a projected rotational velocity $v \sin i=(145 \pm 10) \mathrm{km} \mathrm{s}^{-1}$.

It is generally assumed (Hardorp \& Strittmatter 1970) that all Be stars are fast rotators with approximately the same rotational velocity, the observed velocity spread being due to orientation effects. The largest values of $v \sin i$ measured from line profiles are in the neighbourhood of $400 \mathrm{kms}^{-1}$ (Sletteback 1982). If we assume a true rotational velocity at the equator $v=(400 \pm 50) \mathrm{km} \mathrm{s}^{-1}$, we infer an inclination angle $i=(21 \pm 3) \mathrm{deg}$.

An empirical correlation between the full width at half maximum of the emission component from $\mathrm{H} \alpha$, its equivalent widths and the projected rotational velocity was derived by Dachs et al. (1986):

$$
\begin{aligned}
& \frac{\operatorname{FWHM}(\mathrm{H} \alpha)}{2}\left[\frac{\mathrm{EW}(\mathrm{H} \alpha)}{-3 \AA}\right]^{\frac{1}{4}}-60 \\
& \simeq(v \sin i \pm 30) \mathrm{km} \mathrm{s}^{-1} .
\end{aligned}
$$

In this case, we measure a mean FWHM = $(6 \cdot 1 \pm 0 \cdot 2) \AA=(280 \pm 10) \mathrm{km} \mathrm{s}^{-1}$, and a mean EW $=(-16 \cdot 0 \pm 1 \cdot 0) \AA$ for $\mathrm{H} \alpha$ (Figure 6$)$. This would lead to a projected rotational velocity $v \sin i=(150 \pm 30)$ $\mathrm{km} \mathrm{s}^{-1}$, in agreement with the more reliable value derived from the He I $\lambda 4471$ absorption line.

It is reasonable to assume (Dachs et al. 1986) that the equivalent width of the $\mathrm{H} \alpha$ emission line is proportional to the projected area of the disk orbiting the Be star in the equatorial plane; the disk is made of gas excreted from the star, and its outer radius is expected to increase during an active phase of the system. Using the empirical relation between $\mathrm{H} \alpha$ equivalent width and disk radius given by Dachs et al. (1986), we derive $R_{d}=(3 \cdot 4 \pm 0 \cdot 1) R_{*}$, where $R_{*}$ is the radius of the $\mathrm{Be}$ star and $R_{d}$ is the radius at which optical depth equals unity for $\mathrm{H} \alpha$ emission. As discussed in Section 2.2, we can take $R_{*}=(10 \pm 3) R_{\odot}$ and $M_{*}=(18 \pm 2) M_{\odot}$.
If the circumstellar disk were geometrically thin and in keplerian rotation, the emitting gas at its outer rim would have a projected rotational velocity

$v_{d} \sin i=\left(\frac{G M_{*}}{R_{d}}\right)^{\frac{1}{2}} \sin i=(114 \pm 25) \quad \mathrm{km} \mathrm{s}^{-1}$.

We would therefore expect to observe double-peaked line profiles for $\mathrm{H} \alpha$ and $\mathrm{H} \beta$, with peak-to-peak separations $\simeq 2 v_{d} \sin i \simeq 230 \mathrm{~km} \mathrm{~s}^{-1}($ Smak 1981). This value corresponds to a separation $\Delta \lambda=3 \cdot 7 \AA$ at $\mathrm{H} \beta$, and $\Delta \lambda=5 \cdot 0 \AA$ at $\mathrm{H} \alpha$, well discernible with our $1.2 \AA$ resolution. We observe that both $\mathrm{H} \alpha$ and $\mathrm{H} \beta$ emission line profiles are always symmetrical and single-peaked: this suggests that the circumstellar gas is not confined to a thin disk in the equatorial plane, but may form a thick torus or an envelope which extends to the polar regions of the stellar atmosphere. Alternatively, the absence of double peaks could be due to non-Keplerian motion in the outer disk, where radial outflows can dominate over rotation, or to a much larger disk radius [cf. the model proposed by Poeckert \& Marlborough (1979)]. As shown in Dachs et al. (1986), the $\mathrm{H} \alpha$ emission lines from Be stars are almost always single-peaked for values of $\mathrm{EW}, \mathrm{FWHM}$ and $v \sin i$ similar to those measured in this system.

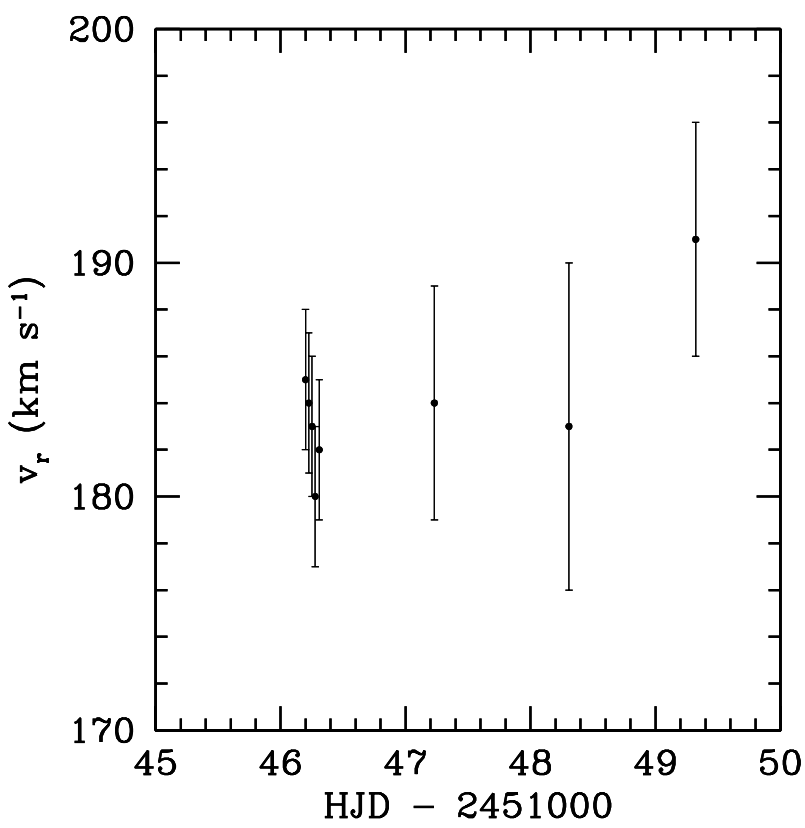

Figure 7-Radial velocity of the optical counterpart of RX J0117 6-7330.

The projected radial velocity of the system was determined by measuring the central position (using a Gaussian fit) of $\mathrm{H} \alpha, \mathrm{H} \beta, \mathrm{He}$ I $\lambda 4388$ and $\mathrm{He}$ I $\lambda 4922$ in each spectrum (two or three consecutive $600 \mathrm{~s}$ spectra were averaged together to increase the $\mathrm{S} / \mathrm{N}$ ); the values found are plotted in Figure 7. Although the variations in the measured radial 
velocity may be due to the orbital motion, the data are insufficient to determine the orbital period or the radial velocity amplitude from these data, or the eccentricity of the orbit (it can be $e \gtrsim 0 \cdot 3$ in Be/X-ray binary systems). All known Be/X-ray binaries have orbital periods $P \gtrsim 15$ days, with periods of hundreds of days in some cases (van den Heuvel \& Rappaport 1987). The average systemic velocity over the time of our spectral observations is $\gamma=(184 \pm 4) \mathrm{km} \mathrm{s}^{-1}$, confirming that the system is located in the SMC, as suggested by Clark et al. (1997).

\section{Conclusions}

We have presented spectroscopic and photometric observations of the optical counterpart of the X-ray transient RX J0117·6-7330 in the Small Magellanic Cloud, currently in an inactive state, as inferred from the All Sky Monitor 2-12 keV light curve. The data allow us to identify the primary star as a B0 5 IIIe star, of mass $M_{*}=(18 \pm 2) M_{\odot}$, radius $R_{*}=(10 \pm 3) R_{\odot}$, effective temperature $T_{\text {eff }}=(2 \cdot 75 \pm 0 \cdot 20) \times 10^{4} \mathrm{~K}$ and bolometric magnitude $M_{\mathrm{bol}}=(-7 \cdot 4 \pm 0 \cdot 2)$ mag. We have derived an inclination angle of the binary system of $i=(21 \pm 3) \mathrm{deg}$ and an average projected radial velocity $\gamma=(184 \pm 4) \mathrm{km} \mathrm{s}^{-1}$; we have also measured the equivalent widths of the main optical lines. Further observations at different orbital phases are necessary to determine the binary period and the mass function of the compact object. Comparison between this set of data and observations taken during an X-ray active state (phase of enhanced mass loss from the Be star) may be useful to determine the orbital parameters of the binary systems, and to improve the current models of mass transfer in HMXBs.

\section{Acknowledgments}

I would like to thank Kinwah $\mathrm{Wu}$, who did some of the photometric observations at the SSO 40 in telescope; thanks also to Stefan Keller and Mike Bessell for their useful comments and suggestions about Be stars.

\section{References}

Bertelli, G., Bressan, A., Chiosi, C., Fagotto, F., \& Nasi, E. 1994, A\&AS, 106, 275

Bessell, M. S. 1993, in Precision Photometry, ed. D. Kilkenny et al. (Cape Town: The Observatory), p. 227

Charles, P. A., Southwell, K. A., \& O'Donoghue, D. 1996, IAU Circ. 6305 (Cambridge: IAU)

Clark, G. W., Remillard, R. A., \& Woo, J. W. 1996, IAU Circ. 6282 (Cambridge: IAU)

Clark, G. W., Remillard, R. A., \& Woo, J. W. 1997, ApJ, 474, L111

Dachs, J., Eichendorf, W., Schleicher, H., Schmidt-Kaler, T., Stift, M., \& Tüg, H. 1981, A\&AS, 43, 427

Dachs, J., Hanuschik, R., Kaiser, D., \& Rohe, D. 1986, A\&A, 159, 276

Feast, M. W. 1991, in The Magellanic Clouds, IAU Symp. 148, ed. R. Haynes \& D. Milne (Dordrecht: Kluwer), p. 1

Hardorp, J., \& Strittmatter, P. A. 1970, in Stellar Rotation, ed. A. Slettebak (Dordrecht: Reidel), p. 48

Kahabka, P., \& Pietsch, W. 1996, A\&A, 312, 919

Poeckert, R., \& Marlborough, J. M. 1979, ApJ, 233, 259

Popper, D. M. 1980, ARA\&A, 18, 115

Slettebak, A. 1982, ApJS, 50, 55

Slettebak, A., Collins, G. W., Boyce, P. B., White, N. M., \& Parkinson, T. D. 1975, ApJS, 29, 137

Smak, J. 1981, Acta Astron., 31, 395

Underhill, A. B., Divan, L., Prévot-Burnichon, M.-L., \& Doazan, V. 1979, MNRAS, 189, 601

van den Heuvel, E. P. J., \& Rappaport, S. 1987, in Physics of Be Stars, ed. A. Slettebak \& T. P. Snow (Cambridge Univ. Press), p. 291

van der Klis, M., et al. 1992, A\&A, 106, 339

van Paradijs, J., \& McClintock, J. E. 1995, in X-ray Binaries, Cambridge Astrophysics Series, ed. W. H. G. Lewin, J. van Paradijs \& E. P. J. van den Heuvel (Cambridge Univ. Press), p. 536 